\title{
Liebe Geotechniker und Freunde des konstruktiven Tiefbaus!
}

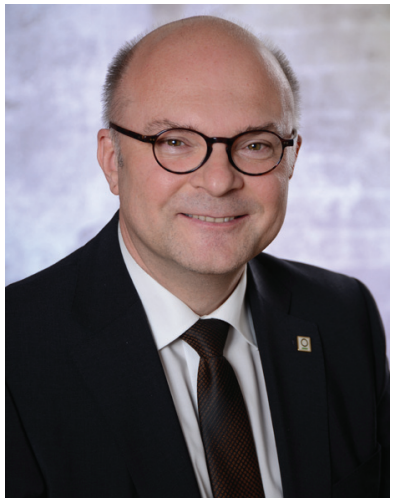

Der Lehrstuhl für Subsurface Engineering der Montanuniversität Leoben, der im Jahr 2014 auf eine 40-jährige Entwicklung zurückblicken konnte, hatte immer das Ziel, das Wissen im Bereich des Bauens in Boden und Fels auf universitäre Basis zu stellen und durch Forschungsarbeiten weiter zu entwickeln. Es freut uns daher, dass wir Ihnen mit der vorliegenden Ausgabe der BHM 12-2015 einen kleinen Einblick in unsere aktuellen Forschungsleistungen, die wir grundsätzlich mit Partnern aus der Industrie und Wirtschaft durchführen, geben können.

Im ersten Aufsatz geben wir Ihnen einen Überblick über die im Oktober 2015 in Kraft gesetzte neue Richtlinie der Österreichischen Bautechnikvereinigung zur Verwendung von Tunnelausbruchmaterial. Dazu ist festzuhalten, dass das Bauwesen zu ca. einem Drittel zum Treibhauspotenzial beiträgt, einen Anteil von rund $40 \%$ des globalen Energiebedarfs und einen Anteil am Abfallaufkommen von über $50 \%$ hat. Tunnelausbruchmaterial und dessen möglichst hochwertige und vollständige Verwertung stellt daher einen wichtigen Beitrag zur Gestaltung eines zukunftsfähigen Wirtschaftssystems durch Steigerung von Effizienz und Effektivität dar.

Seit Ende 2013 wurde gemeinsam mit der ÖBB-Infrastruktur und der Mithilfe der STRABAG und VIGL Consult an der Konzeptionierung und Errichtung eines europaweit einzigartigen Tübbingprüfstandes gearbeitet, der nun im Sep- tember 2015 in Betrieb genommen werden konnte. Es freut uns besonders, dass wir Ihnen heute bereits erste Ergebnisse zur Verifizierung desTrag- und Verformungsverhaltens von Fertigteilbetonsegmenten unter definierten Belastungsbedingungen vorstellen können. Erwähnt sei, dass mit dieser Prüfmaschine nun auch die Möglichkeit der Prüfung von sonstigen Großbauteilen im 1:1 Maßstab besteht.

Eine weitere wesentliche Entwicklung betrifft die Herstellung von Querschlägen im kontinuierlichen Vortrieb. Der kritische Bereich ist hierbei der Übergang zwischenTunnelröhre und Querschlag, da für die Auffahrung des Querschlags der Ringschluss der Tunnelröhre aufgelöst wird. Diesbezüglich wurde unter Heranziehung eines französischen Dübelherstellers auf Initiierung der ÖBB-Infrastruktur AG eine neuartige Verbindungskonstruktion entwickelt. Zusätzlich wurden unterschiedliche Möglichkeiten zur Bestimmung des Bauteilversagens auf Basis der Risserkennung aufgezeigt.

Wesentliche Erkenntnisse zur mechanischen Beständigkeit von Tunnelabdichtungsbahnen im Verbund mit Spritzbeton in Tunnelschalen konnten im Rahmen eines Projektes, bei welchem seit dem Jahr 2009 Materialien von Bestandstunneln auf deren Langzeitbeständigkeit überprüft wurden, gewonnen werden.

Vollkommen neue Erkenntnisse ergaben sich ferner durch Anwendung des Keilspalttests für die Zugfestigkeitsbestimmung von Geomaterialien, der numerischen Simulation des Mini-Disken-Schneidversuchs für Penetrationsprognosen von Tunnelbohrmaschinen und aus der axialen Durchschallung von Prüfkörpern, welche nun erstmals während der Durchführung einaxialer Druckversuche durchgeführt wurde.

Ich wünsche Ihnen eine frohe Weihnachtszeit und Alles Gute für das Neue Jahr 2016!

Ein herzliches Glückauf!

Robert Galler

Leoben, Österreich

E-mail: robert.galler@unileoben.ac.at 